\title{
Multi-scale and multi-physics: towards next-generation engineering materials
}

Published online: 19 May 2020

(C) Springer-Verlag GmbH Germany, part of Springer Nature 2020

\begin{abstract}
Multi-scale and multi-physics are two important thematics when thinking to how next-generation materials will be engineered, aimed at delivering exceptional performances. This special issue attempts at collecting a representative sample of the interdisciplinary efforts that the scientific community is making towards the understanding and exploitation of the complex behaviours that can arise from multi-scale and multi-physics interactions. This contribution aims at contextualising and giving a perspective on the current trends and ideas which drive these efforts.
\end{abstract}

Keywords Multi-scale $\cdot$ Multi-physics

\section{Introduction}

Recent improvements in manufacturing techniques able to manipulate (different) materials $[46,56,62]$ at small length scales are currently pushing the community of theoretical and computational mechanicians towards the development $[3,9,43,92]$ and computation $[1,8,31,37,50,98]$ of reliable mathematical descriptions of engineering multi-physics micro-structured materials. A key observation in this field is that micro-structured materials made up of many elements whose interaction is governed by relatively simple laws can give rise to remarkable and complex collective behaviours [75]. Nevertheless, dealing with many elements by using element-by-element discrete descriptions $[5,97,99]$ can be challenging in different respects. Roughly speaking, when discrete systems are made up of sub-systems which are periodically repeating, and the size of these subsystems is much smaller than the total one, spatially continuous formulations are able to describe the behaviour of the system at large and thus to capture and/or reveal emerging behaviours $[13,39,54,57,58,92]$ Even though available computational capabilities have remarkably increased during the last years, the numerical analysis of discrete systems with many degrees of freedom can still get easily very complex with respect to the equivalent continuum models. In conclusion, a multi-scale approach is particularly suited when studying multi-physics micro-structured materials, provided that one can bridge different scales [11,26,33,80,81] and couple different physics $[32,52,53]$. The different techniques which enable passing from a refined scale to a coarser one can be grouped under the name "homogenisation" [3,6,13,16,30,40,50,54,83,95,102]. Multi-scale and multi-physics are, in short, approaches of utmost importance when dealing with materials whose performances are due to a strong coupling between different scales and physical phenomena, like in the emerging field of metamaterials $[18,29,33-35,42,44,56,66,68,100]$. This special issue attempts at giving an account, as broad as possible, of the interdisciplinary efforts that the scientific community is making towards the understanding and exploitation of the complex behaviours that can arise from multi-scale and multi-physics interactions.

E. Barchiesi $(\varangle)$

International Research Center M\&MoCS, Università degli Studi dell'Aquila, Via Giovanni Gronchi 18 - Zona industriale di Pile, 67100 L'Aquila, Italy

E-mail: BarchiesiEmilio@gmail.com 
Topics include local buckling of composites, wave dispersion in micro-structured solids, phase field modelling of crack growth, isogeometric and assumed strain finite element analysis of composite laminates, boron nitride nanotubes for nano-sensing, electromechanical coupling of Bleustein-Gulyaev wave propagation in prestressed piezoelectric-layered materials, two-temperature generalised magneto-thermoelastic formulations, and Becker-Döring modelling for two-phase materials.

\section{Multi-scale and multi-physics: emerging ideas by examples}

As mentioned above, scale bridging is one of the most relevant challenges in the multi-scale mathematical description of engineering materials and structures. Particularly, discrete-to-continuum bridging, with special emphasis on the identification of standard and enriched kinematical fields in generalised continuum descriptions, is currently a hot topic in the field $[11,26,33,80,81]$. The multi-scale thematic is a transverse one, being the protagonist of many recent developments in digital image correlation techniques. Indeed, aimed at substantiating and improving the mathematical descriptions at different scales, current research work is also directed towards interfacing experimental measurements, obtained by imaging techniques, with numerical modelling $[14,19-24,27,48,59,65,70,77-79,86,87]$ in a scale-consistent-and hence kinematically consistent way, e.g. homogenised continuum modelling is fitted and validated by full-field measurements [63,64,69,76,85, 89,93].

Generalised continuum theories are experiencing a renewed interest from different scientific communities working in mechanics, especially those dealing with metamaterials. Indeed, after the initial impetus given by the early works of the 60's $[35,36,72-74,96]$, up to a few years ago the field of generalised continua was sustained only by some small academic niches. While the use of macroscale continuum descriptions in modelling engineering materials is widespread, it is now well-known that models within the standard Cauchy continuum mechanics framework are not able to properly describe the mechanical behaviour of these materials in presence of strong heterogeneities at micro-scale [51,60,71,97], like in metamaterials. Thanks to their enriched kinematics [49,66,67] and/or non-local features [3,8,9,13,37,51,68,82,83,91], so-called generalised continua are needed to capture micro-scale deformation mechanisms, including long-range interactions [94], which are indeed conferring many sought non-standard macroscopic properties [18].

To get more insight into these phenomenological theories, much ongoing work is aimed at bridging the gap between discrete and continuum. A promising research line is that exploiting asymptotic variational homogenisation techniques $[2,3,10,12,16,40,83,102]$ to transfer the discrete description of a self-similar finite-dimensional system into a continuous formulation, when the degrees of freedom tend to infinity. Indeed, such techniques can deal with discrete systems whose kinematics is such that the emerging continua belong to the class of generalised continua with enriched kinematics. These variational asymptotic homogenisation procedures, used to deduce the continuum, can be generally sketched as follows

(i) A family of discrete spring systems with micro-length scale $\varepsilon>0$ is introduced—generalised coordinates and energy contributions $\mathfrak{E}_{\varepsilon}$ are defined

(ii) The kinematic descriptors of the continuum are introduced as continuous functions-these functions are chosen such that their evaluation at particular points can be related to the generalised coordinates of the micro-model

(iii) Formulation of the deformation energy of the micro-model $\mathfrak{E}_{\varepsilon}$ using the evaluation of the continuum descriptors at particular points, followed by a Taylor expansion of the energy with respect to the micro length scale $\varepsilon$

(iv) Specification of scaling laws for the constitutive parameters in the micro-model followed by a limit process in which the energy of the continuum $\mathfrak{E}$ is related to the micro-model by $\mathfrak{E}=\lim _{\varepsilon \rightarrow 0} \mathfrak{E} \varepsilon$.

\subsection{A recent declination of the multi-scale pantographic motif}

To give a (not outdated!) hint of the potentials offered by these techniques, it may be mentioned that the derivation of a strain gradient 2D-continuum model with three stiffness parameters, being capable of describing arbitrarily large elastic deformations of so-called bi-pantographic fabrics in plane (see Fig. 1), was recently achieved by the procedure sketched above [12,15]. Bi-pantographic fabrics are engineering fabrics controlled by two micro-scales: they are pantographic fabrics $[39,45,46,54,80,81,90]$ whose fibres are in turn pantographs $[13,17]$. The kinematics of the homogenization target is given by its displacement function $\mathbf{u}_{H C}: \Omega \rightarrow \mathbb{R}^{2}$. In Fig. 1, the domain $\Omega$ is the plain, i.e. no holes, rectangle whose sides are $\partial \Omega_{1}, \partial \Omega_{2}, \partial \Omega_{3}$, and $\partial \Omega_{4}$. Bias 


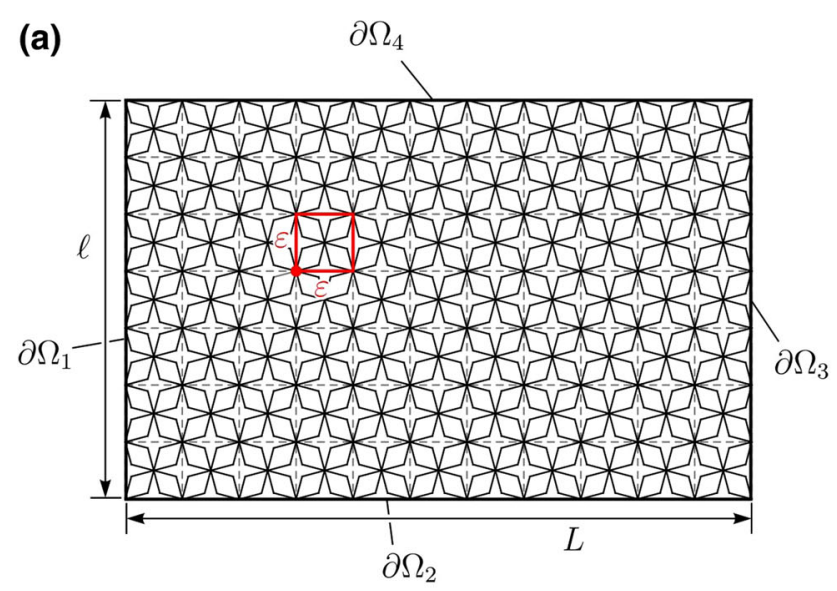

(b)

(c)

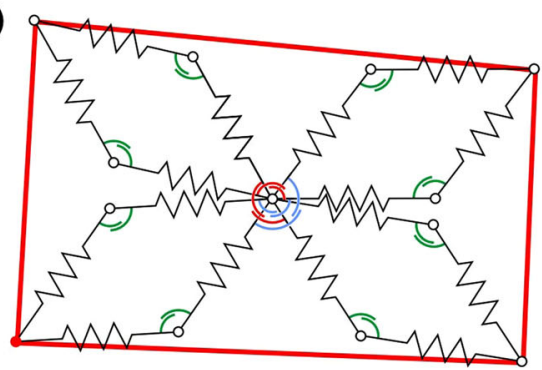

Fig. 1 Bi-pantographic fabrics $(L=187 \mathrm{~mm}, \ell=119 \mathrm{~mm})$. a Domain $\Omega$ b Undeformed configuration of a unit cell $\mathbf{c}$ Force elements and deformed configuration of a unit cell

Table 1 Nominal continuum modelling boundary conditions

\begin{tabular}{ll}
\hline$\partial \Omega_{1}$ & $\partial \Omega_{3}$ \\
\hline $\mathbf{u}_{H C}(\mathbf{x})=0$ & $\mathbf{u}_{H C}(\mathbf{x})=\bar{u} \mathbf{e}_{\zeta}, \bar{u} \in \mathbb{R}^{+}$ \\
{$\left[\nabla \mathbf{u}_{H C}(\mathbf{x})\right] \mathbf{n}(\mathbf{x})=0$} & {$\left[\nabla \mathbf{u}_{H C}(\mathbf{x})\right] \mathbf{n}(\mathbf{x})=0$} \\
\hline
\end{tabular}

The vector $\mathbf{n}$ is the unit normal to $\partial \Omega=\cup_{i=1}^{4} \partial \Omega_{i}$ in $\mathbf{x}$

extension test of bi-pantographic fabrics has been nominally defined through the essential boundary conditions in Table 1. Non-classic kinematics prescriptions in the second row, as combined with those in the first row of Table 1, imply that $\nabla \mathbf{u}_{H C}(\mathbf{x})=0$ on the short sides of the specimen.

Lightweight rectangular bi-pantographic specimens have been additively manufactured by selective laser sintering (SLS) 3D-printing using polyamide $[41,46,56]$. The condition $\nabla \mathbf{u}_{H C}(\mathbf{x})=0$ was realised by introducing stocky rhomboidal elements. Specimens have been subjected to displacement-controlled bias extension tests for total elastic deformations up to ca. $40 \%$ (see Fig. 2). It was observed that bi-pantographic fabrics exhibit an extremely wide elastic range. Such extreme elastic deformations are achievable as the total deformation is considerably greater than single-element deformations. Indeed, elements arrange locally in space in such a way as to minimise the total deformation energy by mimicking mechanisms corresponding to zero-energy deformation modes, a hallmark of second gradient materials. Being pantographic fabrics whose fibres are in turn pantographs, zero-energy deformation modes of bi-pantographic fabrics correspond to combinations of pantographic mechanisms at both scales (see Fig. 3). For total large deformations, in the most deformed regions of the specimen, pantographic mechanisms at said scales are progressively deployed until a densification phenomenon, similar to that occurring in compressed foams, is observed and the material toughens dramatically, undergoing a kind of phase transition.

Research work carried out in the design and modelling of bi-pantographic fabrics is also being complemented by the quantitative validation of homogenised modelling as descriptive and predictive tool. These steps, i.e. design, modelling and validation, should be considered as strongly interconnected in the development of a new metamaterial; they may be repeated multiple times as a feedback loop $[35,101]$. The deformations of the 

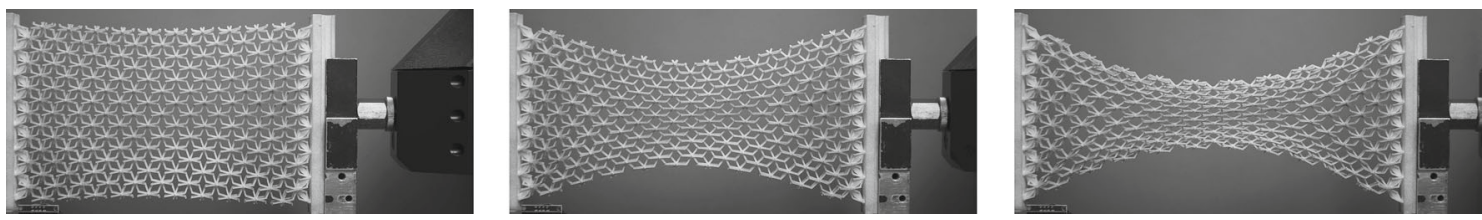

Fig. 2 Bias extension test of bi-pantographic fabrics

(a)
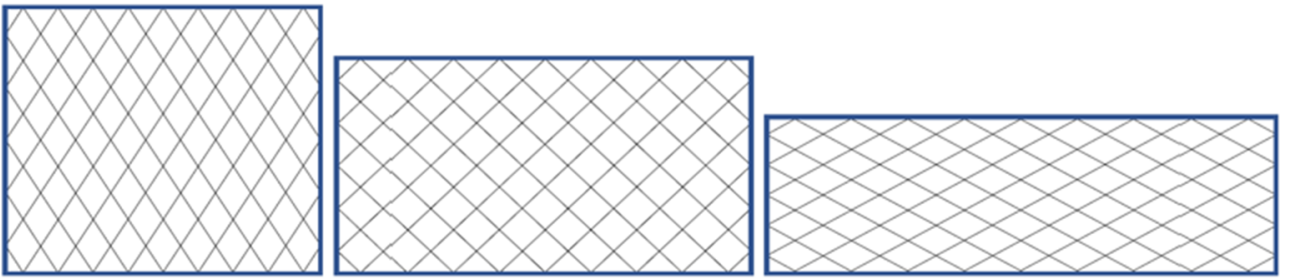

(b)

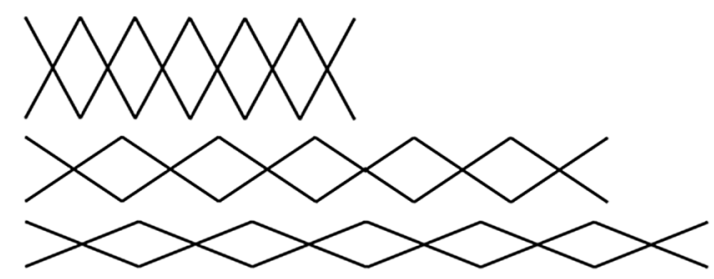

(c)

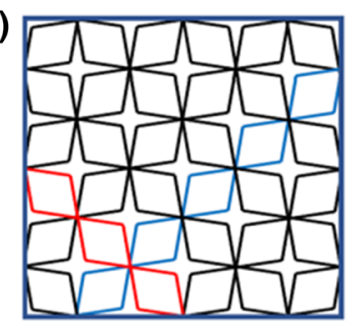

Fig. 3 Uniform shearing floppy modes for pantographic fabrics (a), uniform extension floppy modes for pantographic fibres (b). $\mathrm{Bi}$-pantographic fabrics are pantographic fabrics whose fibres are pantographs (c)

bi-pantographic sample were measured via FE-based digital image correlation (DIC). Clearly, notwithstanding that lower-scale, i.e. local, mechanical and DIC analyses [86,87] considering richer kinematics and geometries could achieve better results in terms of grey-level correlation residuals, the objective is to assess to what extent a coarse macroscale continuous description can represent and predict experimental measures. Aimed at a consistent comparison between modelling and DIC, the two of them shall employ the same (macroscale) domain geometry and kinematics. This motivates the use of global DIC [76], where the underlying hypothesis is the continuity of the displacement field. It is worth to be remarked that such macroscale analyses would clearly compare better with experimental pictures should the number of unit cells be increased, as the scale separation would become more evident. Weak-scale separation implies that the background area fraction within the plain, i.e. no holes, region of interest (ROI) shaped as the whole specimen is non-negligible [25]. Being the background a low-contrast region and not moving, this affects negatively the performance of global DIC, it being an inverse problem with multiple solutions. For the reasons discussed above, a mechanical regularisation of the (inverse) DIC problem must be used as a filtering to recover mechanically admissible displacements. Measured boundary conditions drive the numerical model of the experiment. Indeed, while experimentally it is attempted to enforce displacement boundary conditions in Table 1, displacement at constrained boundaries as measured by DIC is generally different. The grey-level residuals are utilised to independently probe DIC and FE simulations against the full video of the experiment. Given an experimentally registered picture, the grey level correlation residuals, i.e. difference between the current picture and the one obtained by applying the displacement field to the initial picture, are chosen as a point-wise measure of goodness. Its root-mean-square (RMS) computed over the region of interest (ROI) is chosen as a global goodness measure. A flowchart of the analysis is shown for an exemplary picture in Fig. 4. Preliminary results indicate that measured and computed displacements fields seemingly overlap. To have a closer look, the grey-level correlation residuals and their RMS over the ROI are computed for the full experiment. It is observed that in the correlation residuals the micro-scale pattern emerges, which is expectable as it is obviously not completely taken into account at the considered description scale.

Future work shall include the following points, requiring the development of new modelling, computational and experiment-numerics interfacing tools 


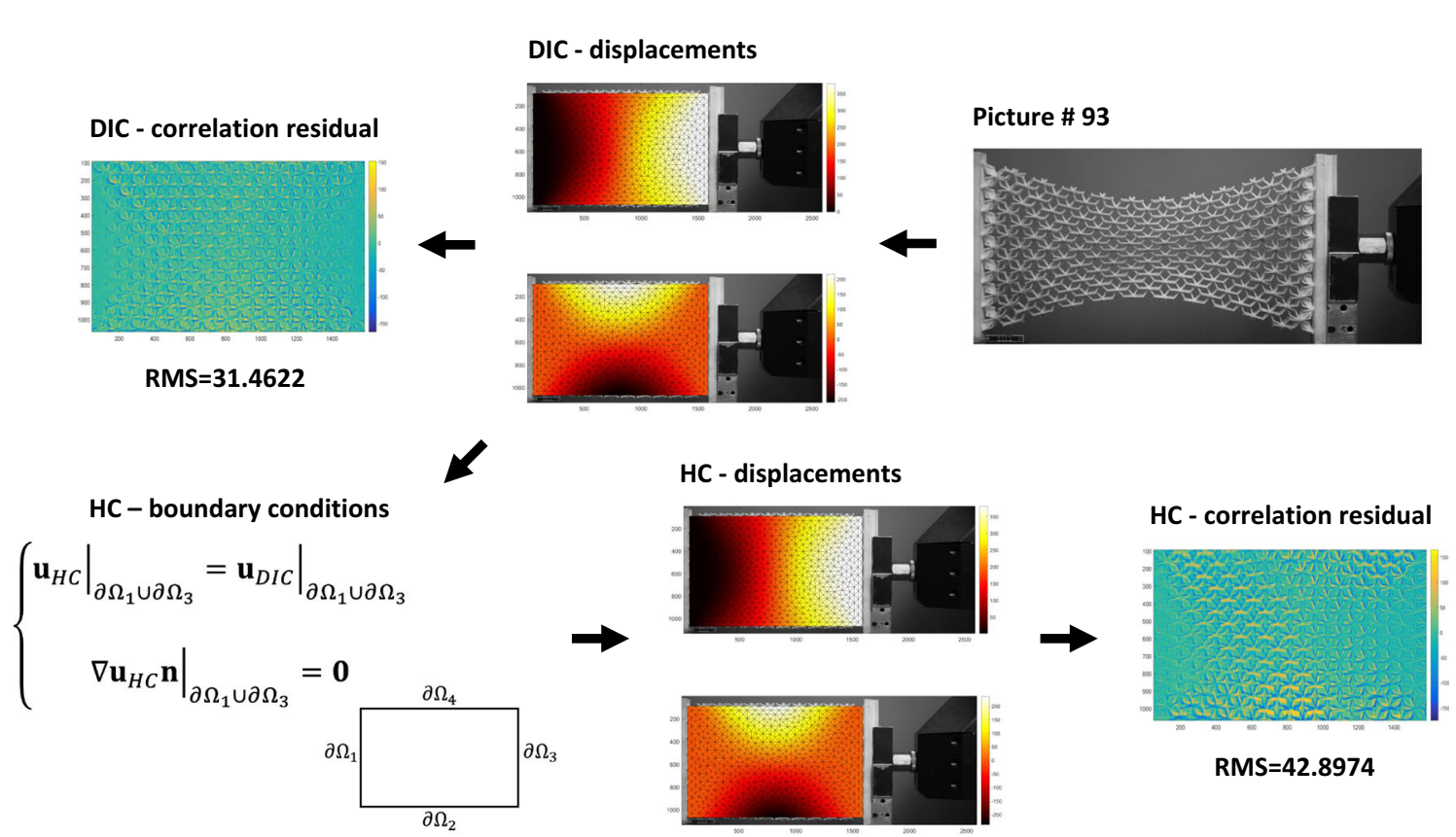

Fig. 4 Flowchart of the process followed by comparing global digital image correlation (DIC) and homogenised continuum (HC) modelling for a given exemplary picture
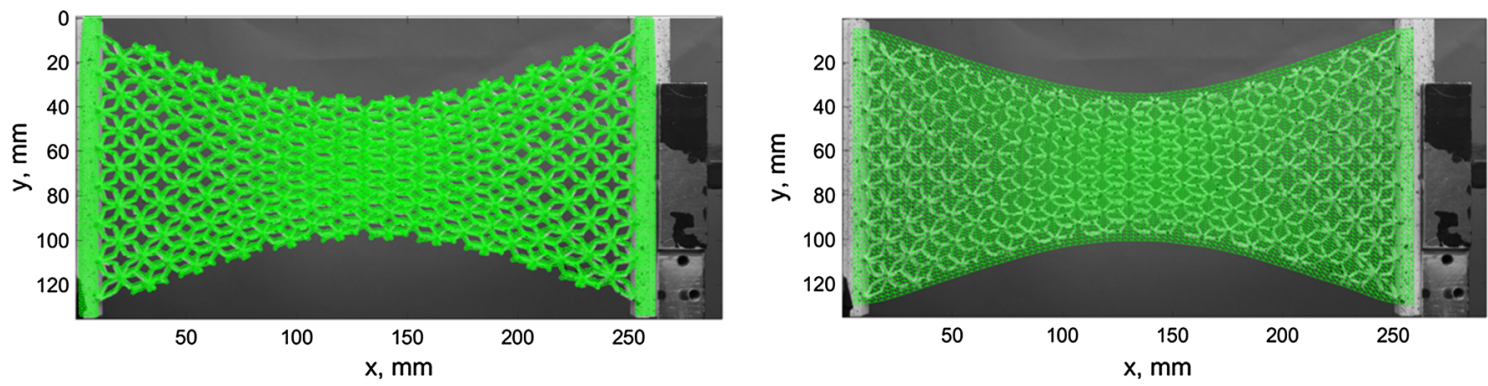

Fig. 5 DIC across different geometries and kinematics for multi-scale analyses: holed domain taking into account bi-pantographic microstructure (continuous kinematics, left), and plain domain (continuous kinematics, right), i.e. global DIC. Courtesy of François Hild

- applying the validation procedure to a wide class of in-plane experiments as bi-axial extension/shearing tests and to different scales (see Fig. 5)

- applying the validation procedure to three-dimensional experimental observations elaborated by Digital Volume Correlation, i.e. full volume displacement and strain field measurements, at different scales (local kinematic fields could be analysed by techniques as X-ray tomography)

- addressing 3D buckling instabilities through generalised plate models derived by homogenisation [8].

To recap, past work in bi-pantographic fabrics has been here presented as an exemplary case, as it dealt with many interdisciplinary aspects of metamaterials' synthesis. It indeed consisted in

- the formulation of a nonlinear discrete Hencky-type spring model of bi-pantographic fabrics at micro-scale and its numerical implementation [9]

- the asymptotic homogenisation of such a discrete system in statics and dynamics regimes by a twostep procedure based on energy limits: a. homogenisation of a discrete pantographic fibre [13,17], $b$. homogenisation of a 2D lattice made by assembling pantographic fibres (see Fig. 3c) leading to a generalised continuum formulation [12]

- the numerical validation of the homogenisation procedure $[13,14]$

- the design and additive manufacturing of bi-pantographic fabrics specimens in Polyamide base material for bias extension test and experimental data collection [12] 

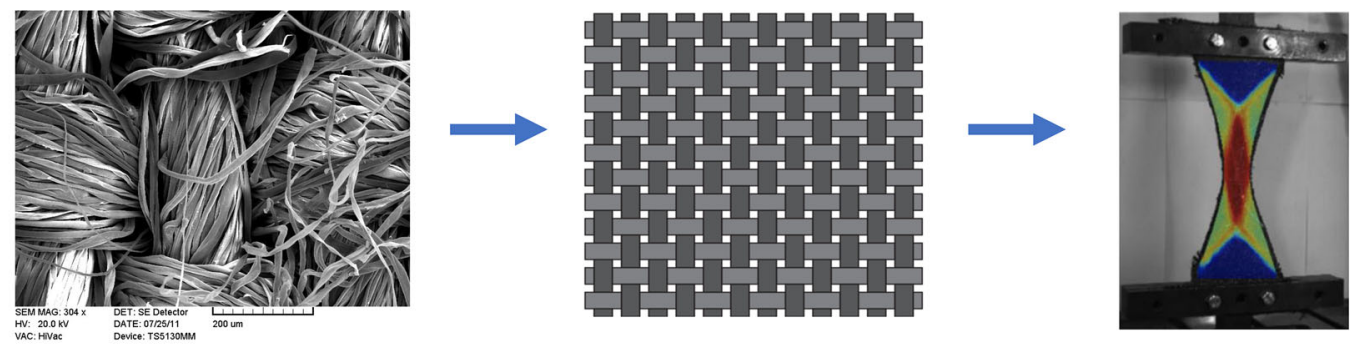

\section{$\uparrow \downarrow$}

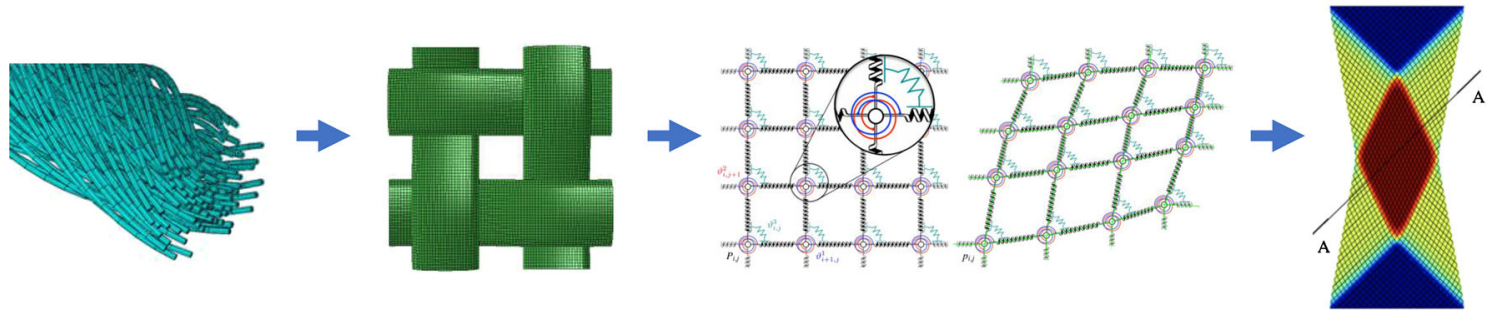

Fig. 6 Multi-scale mathematical description of fibrous materials

- the DIC/numerics interface for discrete and continuous modelling aimed at experimental validation [10, $14,15]$

On the applicative side, previous research allowed for the mathematical description, design, manufacturing and experimentation of fibred metamaterials with an extremely wide elastic range, showing a nonlinear toughening effect for large deformations.

\subsection{Multi-scale mechanics of fibrous engineering materials}

Aimed at further exemplifying the significance of the multi-scale paradigm, a perspective on its use in the description (obviously directed towards the understanding, and design) of ordered fibrous materials with continuous fibres, like those employed in reinforced composites, will be given. Possible future directions will be traced and elucidated. Current research deals indeed with multi-scale descriptions leading to generalised continua at macroscale. The final objective of ongoing research is the identification of standard and enriched kinematical fields, by interfacing numerics and experimental data elaborated by 2D/3D imaging techniques.

In fibrous materials different scales may be identified. A possible choice can be the following one: microscopic scale (fibres), mesoscopic scale (yarns) and macroscopic scale (specimen), see Fig. 6. The microand meso- structures play a crucial role on the overall mechanical behaviour at the macroscopic scale. One key point of the ongoing research in the field is the understanding of the (non-local) properties conferred to the overall material behaviour by complex weaving patterns as, e.g. $2.5 \mathrm{D}$ weaving. Weaving pattern indeed strongly determines the overall material properties: for instance, $2.5 \mathrm{D}$ weaving can be employed in woven reinforcements, where multiple layers of warp are joined together by a plurality of wefts. This structure of interwoven layers avoids the problems of delamination that may occur in multi-layered materials obtained by superimposing independent woven layers, and permits the realisation of thick reinforcements. Such materials are reserved for advanced aeronautics and aerospace applications.

As mentioned above, when applied to heterogeneous fibrous materials at the specimen scale, conventional continuum modelling techniques fail to capture the complex phenomena occurring across multiple length scales [88]. Therefore, a multi-scale modelling approach must be devised to pass information across the different length scales [47,50]. Macroscale modelling is thus required to be able to catch the macroscopic effects due to meso-scale phenomena and properties. Owing to the geometric arrangement of the two families of yarns, orthotropic behaviour must be accounted for at macroscale [80,81]. Yarns are constituted by many fibres. When a yarn is subjected to tension, a linear behaviour can be identified if it is made by elastic and non-twisted or non-braided fibres. Given the relatively high tension stiffness, modelling may be based on the hypothesis that 
yarns are inextensible. More complex constitutive laws could be then conceived extending such a limit case. Nevertheless, such hypothesis introduces some difficulties: indeed, the ratio between the extensional stiffness and the shearing stiffness would go to infinity. A further mechanism which should be considered as having an effect on the macroscale behaviour of fibrous materials is the compaction of yarns. It is defined as their cross-section area change. When a yarn is compressed in a direction which is orthogonal to its centre line, then its fibres become more closely packed and fill the voids initially present in the cross section, thus increasing yarns' stiffness. Clearly, such a compaction phenomenon presents an asymptotic behaviour, following an initial phase where fibres move and fill the voids, compaction slows down. Characterising experimentally compaction is currently a challenge. Attempts in this regard shall be made, e.g. by synchrotron X-ray tomography.

Distortion, i.e. cross section deforming without compaction, of yarns is an additional deformation mechanism which occurs at the meso-scale. For instance, one can consider a yarn subjected to a bending deformation: fibres are forced to slide one with respect to the other in order to let the yarn assume the desired form and, at the same time, keep the fibres' quasi-inextensibility constraint. This internal sliding of fibres can be interpreted as a motion of the yarns' cross sections (for example a rotation). Yet another deforming mechanism can be identified for yarns which is transverse shearing. It consists in fibre-fibre sliding in the direction of the fibres themselves. It is worth noting that a coupling mechanism can be recognised between the compaction of a yarn and its distortion: when the yarn is compacted, its distortion occurs with increased difficulty. Indeed, when fibres are compacted then friction mechanisms are more pronounced, which renders sliding more difficult. For the same reason it is also clear that, as for the distortion, an increased compaction of a yarn causes a stiffening effect on its shearing. As yarn shearing and distortion deformations are affected by the same microscopic mechanisms at fibres' level, a coupling exists between them. Yarns' bending is another relevant meso-scale deformation mechanism to be taken into account. All these mechanisms are neglected in Cauchy modelling and this determines its failure in describing and predicting the macroscopic behaviour of fibrous materials $[28,38,61]$. Aimed at modelling, the meso-scale features mentioned above, yarns may be modelled as generalised nonlinear 1D continua with an enriched kinematics [7]. Applying the homogenisation procedure sketched at the beginning of this article to a lattice consisting in two orthogonal families of such 1D continua, one would clearly obtain a generalised 2D continuum with enriched kinematics. As two touching yarns are kinematically free to move one with respect to the other, the target continuum shall have two displacement fields, i.e. one for each family of yarns, thus being a mixture theory. Clearly, a conservative or non-conservative coupling should be introduced between the two displacements to account for the sliding friction (in addition to the coupling between two orthogonal yarns' rotations). In fact, assuming that two touching yarns can rotate around their contact point with no slipping, thus implying a zero relative displacement among the two families of yarns, is generally reasonable. Indeed, during experimental analyses-even at finite strains-when straight lines are drawn on textile reinforcements, these lines become curved still remaining continuous. It is foreseeable that simplifying kinematic assumptions should be able to recover, as a special case, a second gradient theory $[2,55]$. The rigorous derivation by homogenisation and utilisation of second gradient theories in the modelling of ordered fibrous materials has been already widely studied in the literature [4,5]. Even representing a special case, such theories are still capable of describing relevant properties, as the formation of finite-size shear boundary layers due to fibres bending [37,98]. Second gradient theories are indeed able to account for the macroscopic manifestation of the mesoscopic bending of the yarns. It is worth to mention that second gradient theories are also able to catch local size effects, manifesting as shear bands, which are ultimately due to nonlinearities in the two main micro-scale deformation mechanisms of yarns, i.e. fibre-scale transverse compression and shearing.

Experimental methodologies for the quantitative analysis of deformation mechanisms occurring at the fibre scale play an important role in this research. Image processing tools to analyse 3D images of evolving fibrous microstructures obtained by X-ray microtomography are being developed $[10-13,15]$. These techniques are applicable even in composite materials with an optically opaque matrix. These tools further allow for the tracking of position, orientation, displacement and deformation (bending, twisting, shearing, extension/compression, distorsion) of each fibre together with the position and the evolution of each fibre-fibre contact. The in situ tracking of these microstructure descriptors provides useful information for theoretical or numerical models.

To recap, current research in fibrous materials aims at a full expedition towards the macroscopic modelling of fibrous materials by means of generalised continua, possibly including damage phenomena, with applications in biomimetic materials like arterial tissues and composites reinforced with architectured fibrous networks [14]. Investigations are carried out from experimental characterisation and modelling at micro-scale all the way up to macroscopic modelling and validation by imaging/numerics interfacing. Discrete/finite element simulations 


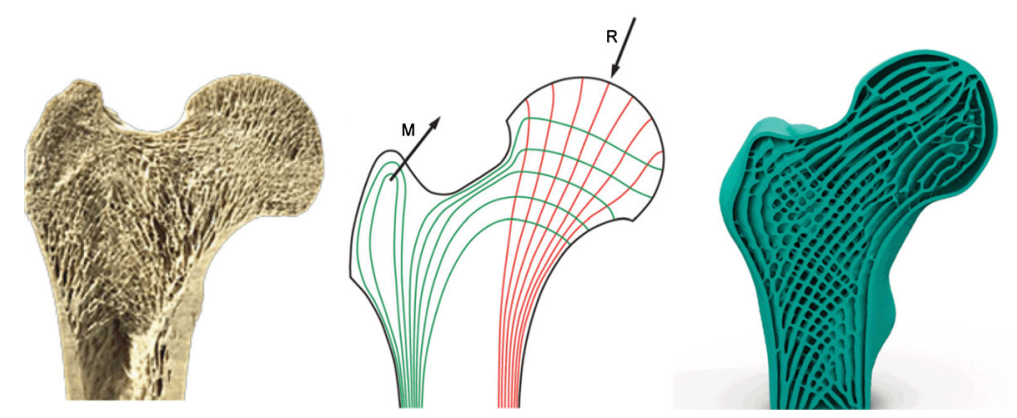

Fig. 7 From bone to additively manufactured bio-inspired micro-structured materials through topological optimisation
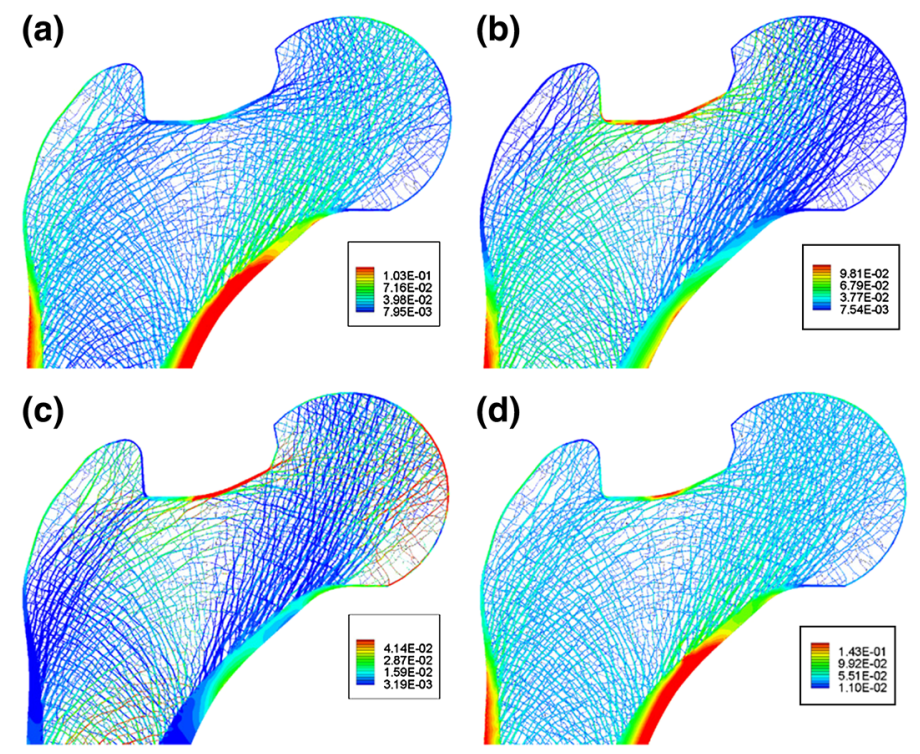

Fig. 8 Topological optimisation results in natural bone microstructure

performed at the fibre scale are a relevant tool to get a better understanding of the micro-scale, and benefit from the availability of experimental data collected at the fibre scale.

\subsection{Conception of multi-scale bio-inspired metamaterials}

Among the many declinations that the multi-scale thematic has assumed in the last years, a very promising one regards the conception and manufacturing of bio-inspired micro-structured materials (see Fig. 7). It is well-known that topology optimisation [84] can give many hints on the rational behind biological materials, which are indeed the result of a millenary optimal adaptation process. For example, it has been widely proved that bone is an optimal structure [55], where strength is maximised using the optimal distribution of bone mass (Wolff's law). One of the most appropriate mathematical validations of this law is indeed a structural optimisation-based formulation where total strain energy is minimised against a mass and a space constraint, see Fig. 8. Inspired by this observation, the main question that is currently addressed in facing the conception and design of bio-inspired micro-structured materials is the following one: does there exist (and what is it) the (topology) optimisation problem formulation (design variables, objective function, constraints, loading conditions, etc.) which results in mathematically optimal solutions resembling biological materials? For this purpose, an efficient structural optimisation-based computational model should be devised to be used as a routine within an iterative methodology aimed at solving this complex inverse problem. 

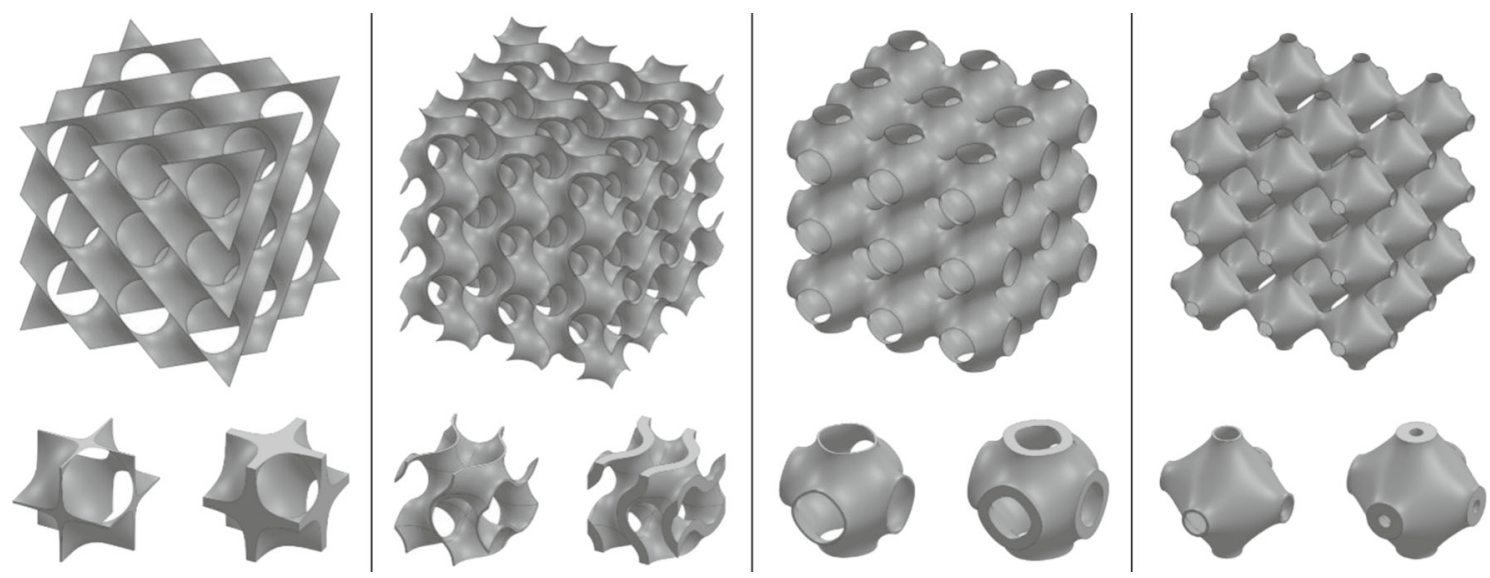

Fig. 9 Lattices based on triply periodic minimal surfaces

\subsection{Multi-scale and multi-physics: metamaterials for comfort in buildings}

Finally, a hint of the significance of the multi-scale and multi-physics thematics in ongoing civil engineering research shall now be given, with regard to the conception, realisation and modelling of innovative highperformance architected materials for comfort in buildings. Some future research perspectives that can be envisaged will be also out-lined.

Energy saving has become one of the most important problems that modern society is facing. Buildings are among the largest consumers of energy. This has attracted the interest of the Civil Engineering community towards their energetically rational design. The issue of material selection in achieving overall energy efficiency is increasingly gaining importance. There is unanimous agreement on the fact that buildings should be designed and constructed in such a way that they consume a minimal amount of energy, but with the simultaneous provision of maximal living comfort. Comfort is affected by many parameters, such as temperature, humidity, air movement, air quality, lighting and noise which are not only dependent upon building architecture and environment, but also on the properties of building materials. The use of high-performance building materials for comfort in buildings is especially recommended, because they are passive elements, thus not requiring external behavioural guidance or energy to operate.

The heat flow through building materials can be explained in terms of their heat capacity and of heat conduction and convection occurring through them. Both heat and moisture might be stored or transmitted through a building material, depending on its hygrothermal properties. An uncontrolled increase of humidity within a building material is undesired; it may cause changes in its thermal, mechanical and sound propagation properties, thus affecting its durability, not to mention that it may trigger or sustain corrosion phenomena. Dry stagnant gas is one of the best insulating materials. The insulating properties of the most successfully employed materials, like closed-cell foams, are determined by the amount of gas held inside the material and by the number of gas pockets. Indeed, air's thermal conductivity can be neglected without affecting the effective thermal conductivity, i.e. the thermal conductivity of the matrix material is for most materials several orders of magnitude higher than the air's one. Therefore, the higher the number of cells (which can maintain the gas stagnant) and the smaller their size, the lower the thermal conductivity of such insulating material. These cells should not be interlinked, as this will allow convection of heat. Indeed, in these materials, heat transport by radiation is often predominant. Also, an optimal thermally insulating building material should be lightweight, yet mechanically stable, so it will not crumble or pack down.

Aimed at increasing energy efficiency and environmental comfort in buildings, the design of innovative architected insulating materials shall take into account the physical picture depicted above. Clearly, those specifications are competing (lightweight vs stiff, etc.) and an optimal trade-off shall be found. Architected materials, as a novel class of low-density materials, gain their multifunctional performances mainly from the underlying periodic architecture, as opposed to the base material. Given a base material, tuning cell shape and geometrical dimensions for fixed mass (iso-mass) or volume (iso-volume) allows to finely control features like mechanical stiffness, interconnectivity, tortuosity, thermal conduction anisotropy, mechanical anisotropy, surface to volume ratio, which are all relevant to the specifications mentioned above for thermally insulating building materials (Fig. 9). 


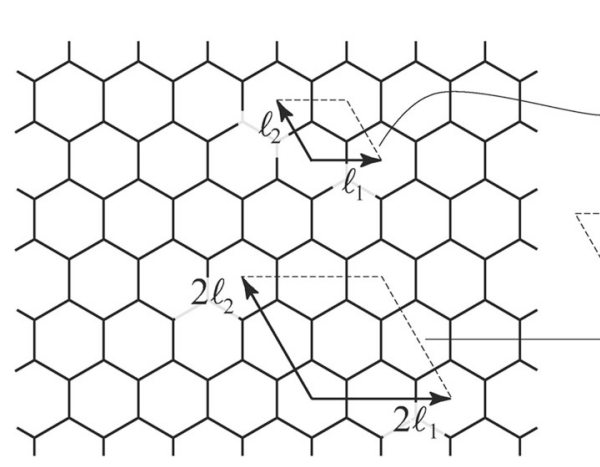

(a) Hexagonal honeycomb

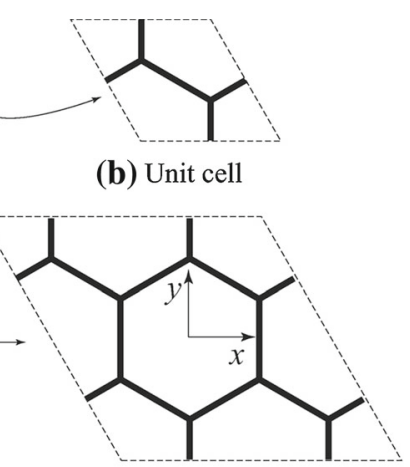

(c) Representative volume element $(2 \times 2$ cells $)$

Fig. 10 Honeycomb structure

Architected materials thus provide design flexibility, which is essential for the optimisation of said competing design targets. One key objective of the ongoing research in the field is the understanding of mechanical and hygrothermal properties conferred to the overall material behaviour by complex material architecture patterns. Indeed, in order to optimise the architected micro-structure of thermally insulating building materials, it is relevant to dispose of computationally efficient macroscopic laws that allow to take into account the heterogeneity and multi-physical complexity of these materials at the micro-scale $[32,53]$. One possible solution to describe materials which are highly heterogeneous on the micro-scale is to assimilate them to equivalent continuous media on a macroscopic scale by using homogenisation methods. The effective medium is then characterised by effective transport and mechanical properties reflecting physics at the architecture scale. Mathematical descriptions at all scales shall couple deformation phenomena with heat, air, and moisture transfer. Most likely, the theory of unsaturated mixture media with heat diffusion shall be exploited at the macroscale. As mentioned above, optimisation over the space of design variables shall be carried out. The effective mechanical properties, as well as hydraulic and heat conductivity for an equivalent continuum, as defined in terms of the material architecture and base material, shall be investigated for any type of architecture-induced heterogeneity. Studies shall be performed to assess the role of humidity transfer on the material durability and on its thermal behaviour when submitted to environmental solicitations. Potentially interesting architectures include periodic (hierarchical) honeycomb (easily producible industrially) and lattices based on triply periodic minimal surfaces, whose utilisation in structural sandwich panels or core fillers, for which the thermo-mechanical performance is of paramount importance, is promising. It could be relevant to evaluate the microscopic behaviour of such architected materials under hydric solicitations to better understand the swelling induced by relative humidity variations, which is also affected by their multi-scale, anisotropic, and heterogeneous structure. The use of microscopic non-destructive methods like X-ray tomography and Digital Volume Correlation shall be considered as a way to perform hygromechanical and morphological characterisations at the micro-scale (Fig. 10).

The use of bio-based materials, like cellulose and bio-polymers, may be considered in view of the efforts currently made in delivering environmentally sustainable materials not coming from non-renewable sources.

Authors must disclose all relationships or interests that could have direct or potential influence or impart bias on the work:

Compliance with ethical standards

Conflict of interest The authors declare that they have no conflict of interest.

\section{References}

1. Abali, B., Müller, W., Eremeyev, V.: Strain gradient elasticity with geometric nonlinearities and its computational evaluation. Mech. Adv. Mater. Mod. Process. 1(1), 4 (2015)

2. Abdoul-Anziz, H., Seppecher, P.: Strain gradient and generalized continua obtained by homogenizing frame lattices. Mathematics and Mechanics of Complex Systems (2018) 
3. Alibert, J.J., Della Corte, A.: Second-gradient continua as homogenized limit of pantographic microstructured plates: a rigorous proof. Zeitschrift für angewandte Mathematik und Physik 66(5), 2855-2870 (2015)

4. Alibert, J.J., Della Corte, A., Giorgio, I., Battista, A.: Extensional elastica in large deformation as gamma-limit of a discrete 1d mechanical system. Z. Angew. Math. Phys. 68(2), 42 (2017)

5. Alibert, J.J., Della Corte, A., Seppecher, P.: Convergence of hencky-type discrete beam model to euler inextensible elastica in large deformation: rigorous proof, pp. 1-12 (2017)

6. Allaire, G.: Homogenization and two-scale convergence. SIAM J. Math. Anal. 23(6), 1482-1518 (1992)

7. Altenbach, H., Bîrsan, M., Eremeyev, V.A.: Cosserat-type rods. In: Generalized Continua from the Theory to Engineering Applications, pp. 179-248. Springer (2013)

8. Andreaus, U., dell'Isola, F., Giorgio, I., Placidi, L., Lekszycki, T., Rizzi, N.: Numerical simulations of classical problems in two-dimensional (non) linear second gradient elasticity. Int. J. Eng. Sci. 108, 34-50 (2016)

9. Auffray, N., dell'Isola, F., Eremeyev, V., Madeo, A., Rosi, G.: Analytical continuum mechanics à la Hamilton-Piola least action principle for second gradient continua and capillary fluids. Math. Mech. Solids 20(4), 375-417 (2015)

10. Barchiesi, E., dell'Isola, F., Hild, F., Seppecher, P.: Two-dimensional continua capable of large elastic extension in two independent directions: asymptotic homogenization, numerical simulations and experimental evidence. Mech. Res. Commun. 103, $103466(2020)$

11. Barchiesi, E., dell'Isola, F., Laudato, M., Placidi, L., Seppecher, P.: A 1D continuum model for beams with pantographic microstructure: Asymptotic micro-macro identification and numerical results. In: Advances in Mechanics of Microstructured Media and Structures, pp. 43-74. Springer (2018)

12. Barchiesi, E., Eugster, S.R., dell'Isola, F., Hild, F.: Large in-plane elastic deformations of bi-pantographic fabrics: asymptotic homogenization and experimental validation. Math. Mech. Solids 25(3), 739-767 (2020)

13. Barchiesi, E., Eugster, S.R., Placidi, L., dell'Isola, F.: Pantographic beam: a complete second gradient 1d-continuum in plane. Zeitschrift für angewandte Mathematik und Physik 70(5), 135 (2019)

14. Barchiesi, E., Ganzosch, G., Liebold, C., Placidi, L., Grygoruk, R., Müller, W.H.: Out-of-plane buckling of pantographic fabrics in displacement-controlled shear tests: experimental results and model validation. Continuum Mech. Thermodyn. 31(1), 33-45 (2019)

15. Barchiesi, E., Hild, F., dell'Isola, F.: On the validation of homogenized modelling for bi-pantographic metamaterials via digital image correlation. International Journal of Solids and Structures. (submitted)

16. Barchiesi, E., Khakalo, S.: Variational asymptotic homogenization of beam-like square lattice structures. In: Mathematics and Mechanics of Solids, p. 1081286519843155 (2019)

17. Barchiesi, E., Laudato, M., Di Cosmo, F.: Wave dispersion in non-linear pantographic beams. Mech. Res. Commun. 94, 128-132 (2018)

18. Barchiesi, E., Spagnuolo, M., Placidi, L.: Mechanical metamaterials: a state of the art. Math. Mech. Solids 5, 967 (2018)

19. Beaubier, B., Dufour, J., Hild, F., Roux, S., Lavernhe-Taillard, S., Lavernhe-Taillard, K.: CAD-based calibration of a 3D-DIC system: principle and application on test and industrial parts. Exp. Mech. 54(3), 329-341 (2014)

20. Beaubier, B., Lavernhe-Taillard, K., Besnard, G., Lavernhe, S., Hild, F., Roux, S.: Cad-based calibration of a 3d dic system. In: Jin, H., et al. (eds.) SEM Conference, vol. 3, pp. 227-232. Springer, (2013)

21. Berke, R., Lambros, J.: Ultraviolet digital image correlation (UV-DIC) for high temperature applications. Rev. Sci. Instrum. 85(4), $045121(2014)$

22. Berny, M., Archer, T., Mavel, A., Beauchêne, P., Roux, S., Hild, F.: On the analysis of heat haze effects with spacetime DIC. Opt. Lasers Eng. 40, 544-556 (2018). https://doi.org/10.1016/j.optlaseng.2018.06.004

23. Berny, M., Jailin, C., Bouterf, A., Hild, F., Roux, S.: Model Reduction for Space-Time DIC. Measu. Sci. Technol. (submitted) (2018)

24. Besnard, G., Guérard, S., Roux, S., Hild, F.: A space-time approach in digital image correlation: movie-DIC. Opt. Lasers Eng. 49, 71-81 (2011)

25. Blazy, J., Marie-Louise, A., Forest, S., Chastel, Y., Pineau, A., Awade, A., Grolleron, C., Moussy, F.: Deformation and fracture of aluminium foams under proportional and non proportional multi-axial loading: Statistical analysis and size effect. Int. J. Mech. Sci. 46, 217-244 (2004)

26. Boutin, C., Giorgio, I., Placidi, L., et al.: Linear pantographic sheets: asymptotic micro-macro models identification. Math. Mech. Complex Syst. 5(2), 127-162 (2017)

27. Calloch, S., Bouvet, C., Hild, F., Doudard, C., Lexcellent, C.: Analysis of mechanical behavior and in situ observations of cu-al-be sma under biaxial compressive tests using dic. In: Proceedings of SPIE, vol. 4537, pp. 83-86. (2002)

28. Cao, J., Akkerman, R., Boisse, P., Chen, J., et al.: Characterization of mechanical behavior of woven fabrics: experimental methods and benchmark results. Compos. A Appl. Sci. Manuf. 39(6), 1037-1053 (2008)

29. Carcaterra, A., dell'Isola, F., Esposito, R., Pulvirenti, M.: Macroscopic description of microscopically strongly inhomogeneous systems: A mathematical basis for the synthesis of higher gradients metamaterials. Arch. Ration. Mech. Anal. 218(3), 1239-1262 (2015)

30. Contrafatto, L., Cuomo, M., Gazzo, S.: A concrete homogenisation technique at meso-scale level accounting for damaging behaviour of cement paste and aggregates. Comput. Struct. 173, 1-18 (2016)

31. Contrafatto, L., Cuomo, M., Greco, L.: Meso-scale simulation of concrete multiaxial behaviour. In: European Journal of Environmental and Civil Engineering, pp. 1-16 (2016)

32. D'Annibale, F., Rosi, G., Luongo, A.: Linear stability of piezoelectric-controlled discrete mechanical systems under nonconservative positional forces. Meccanica 50(3), 825-839 (2015)

33. De Angelo, M., Placidi, L., Nejadsadeghi, N., Misra, A.: Non-standard timoshenko beam model for chiral metamaterial: identification of stiffness parameters. Mech. Res. Commun. 103, 103462 (2020)

34. Del Vescovo, D., Giorgio, I.: Dynamic problems for metamaterials: review of existing models and ideas for further research. Int. J. Eng. Sci. 80, 153-172 (2014)

35. dell'Isola, F., Bucci, S., Battista, A.: Against the fragmentation of knowledge: The power of multidisciplinary research for the design of metamaterials. Zeitschrift für angewandte Mathematik und Physik 67(4) (2016) 
36. dell'Isola, F., Corte, A.D., Giorgio, I.: Higher-gradient continua: the legacy of piola, mindlin, sedov and toupin and some future research perspectives. Math. Mech. Solids 22(4), 852-872 (2017)

37. dell'Isola, F., Cuomo, M., Greco, L., Della Corte, A.: Bias extension test for pantographic sheets: numerical simulations based on second gradient shear energies. In: Journal of Engineering Mathematics, pp. 1-31 (2016)

38. dell'Isola, F., d'Agostino, M., Madeo, A., Boisse, P., Steigmann, D.: Minimization of shear energy in two dimensional continua with two orthogonal families of inextensible fibers: the case of standard bias extension test. J. Elast. 122(2), 131-155 (2016)

39. dell'Isola, F., Della Corte, A., Greco, L., Luongo, A.: Plane bias extension test for a continuum with two inextensible families of fibers: a variational treatment with lagrange multipliers and a perturbation solution. Int. J. Solids Struct. 81, $1-12(2016)$

40. dell'Isola, F., Giorgio, I., Pawlikowski, M., Rizzi, N.L.: Large deformations of planar extensible beams and pantographic lattices: heuristic homogenization, experimental and numerical examples of equilibrium. Proc. R. Soc. A 472(2185) (2016)

41. dell'Isola, F., Giorgio, I., Placidi, L., Spagnuolo, M., Peyre, P., Dupuy, C., Dirrenberger, J., Pawlikowski, M., Igumnov, L.A.: Pantographic metamaterials: a view towards applications (2019)

42. dell'Isola, F., Lekszycki, T., Pawlikowski, M., Grygoruk, R., Greco, L.: Designing a light fabric metamaterial being highly macroscopically tough under directional extension: first experimental evidence. Zeitschrift für angewandte Mathematik und Physik 66, 3473-3498 (2015)

43. dell'Isola, F., Madeo, A., Seppecher, P.: Cauchy tetrahedron argument applied to higher contact interactions. Arch. Ration. Mech. Anal. 219(3), 1305-1341 (2016)

44. dell'Isola, F., Seppecher, P., Alibert, J.J., Lekszycki, T., Grygoruk, R., Pawlikowski, M., Steigmann, D., Giorgio, I., Andreaus, U., Turco, E., Golaszewski, M., Rizzi, N., Boutin, C., Eremeyev, V., Misra, A., Placidi, L., Barchiesi, E., Greco, L., Cuomo, M., Cazzani, A., Corte, A., Battista, A., Scerrato, D., Zurba Eremeeva, I., Rahali, Y., Ganghoffer, J.F., Müller, W., Ganzosch, G., Spagnuolo, M., Pfaff, A., Barcz, K., Hoschke, K., Neggers, J., Hild, F.: Pantographic metamaterials: an example of mathematically- driven design and of its technological challenges. Cont. Mech. Thermod. (2019). https://doi.org/10.1007/ s00161-018-0689-8

45. dell'Isola, F., Seppecher, P., Alibert, J.J., Lekszycki, T., Grygoruk, R., Pawlikowski, M., Steigmann, D., Giorgio, I., Andreaus, U., Turco, E., et al.: Pantographic metamaterials: an example of mathematically driven design and of its technological challenges. Continuum Mech. Thermodyn. 31(4), 851-884 (2019)

46. dell'Isola, F., Seppecher, P., Spagnuolo, M., Barchiesi, E., Hild, F., Lekszycki, T., Giorgio, I., Placidi, L., Andreaus, U., Cuomo, M., et al.: Advances in pantographic structures: design, manufacturing, models, experiments and image analyses. In: Continuum Mechanics and Thermodynamics, pp. 1-52 (2019)

47. Denoual, C., Hild, F.: Dynamic fragmentation of brittle solids: A multi-scale model. Eur. J. Mech. A/Solids 21(1), 105-120 (2002)

48. Dufour, J., Beaubier, B., Lavernhe-Taillard, K., Roux, S., Hild, F.: Shape measurements using cad-based stereodic. In: Proulx, T. (ed.) SEM conference. Springer, (2013)

49. Forest, S., Sievert, R.: Nonlinear microstrain theories. International Journal of Solids and Structures 43(24), 7224-7245 (2006). https://doi.org/10.1016/j.ijsolstr.2006.05.012. http://www.sciencedirect.com/science/article/pii/ S0020768306001703. Size-dependent Mechanics of Materials

50. Geers, M., Kouznetsova, V., Brekelmans, W.: Multi-scale computational homogenization: trends \& challenges. Jnl. Comp. Appl. Math. 234, 2175-2182 (2010)

51. Giorgio, I.: Numerical identification procedure between a micro-cauchy model and a macro-second gradient model for planar pantographic structures. Zeitschrift für angewandte Mathematik und Physik 67(4), 95 (2016)

52. Giorgio, I., Culla, A., Del Vescovo, D.: Multimode vibration control using several piezoelectric transducers shunted with a multiterminal network. Arch. Appl. Mech. 79(9), 859 (2009)

53. Giorgio, I., Galantucci, L., Della Corte, A., Del Vescovo, D.: Piezo-electromechanical smart materials with distributed arrays of piezoelectric transducers: current and upcoming applications. Int. J. Appl. Electromag. Mech. 47(4), 1051-1084 (2015)

54. Giorgio, I., Rizzi, N., Turco, E.: Continuum modelling of pantographic sheets for out-of-plane bifurcation and vibrational analysis. Proc. R. Soc. A Math. Phys. Eng. Sci. 473(2207), 20170636 (2017)

55. Goda, I., Assidi, M., Ganghoffer, J.: A 3D elastic micropolar model of vertebral trabecular bone from lattice homogenization of the bone microstructure. Biomech. Model. Mechanobiol. 13, 53-83 (2014)

56. Golaszewski, M., Grygoruk, R., Giorgio, I., Laudato, M., Di Cosmo, F.: Metamaterials with relative displacements in their microstructure: technological challenges in $3 \mathrm{~d}$ printing, experiments and numerical predictions. Continuum Mechanics and Thermodynamics, pp. 1-20 (2018)

57. Green, A.: Micro-materials and multipolar continuum mechanics. Int. J. Eng. Sci. 3(5), 533-537 (1965)

58. Hild, F.: Relations entre descriptions probabiliste et continue du comportement mécanique de composites à matrice fragile. Rev. Comput. Math. Avd. 8(9-22) (1998)

59. Hild, F., Roux, S.: Measuring stress intensity factors with a camera: Integrated digital image correlation (i-dic). C. R. Mécanique 334, 8-12 (2006)

60. dell Isola, F., Seppecher, P., Della Corte, A.: The postulations á la d'alembert and á la cauchy for higher gradient continuum theories are equivalent: a review of existing results. Proc. R. Soc. A, 471. The Royal Society (2015)

61. Khan, M., Mabrouki, T., Vidal-Salle, E., Boisse, P.: Numerical and experimental analyses of woven composite reinforcement forming using a hypoelastic behaviour. application to the double dome benchmark. J. Math. Proc. Technol. 210(2), 378-388 (2010)

62. Koch, F., Lehr, D., Schönbrodt, O., Glaser, T., Fechner, R., Frost, F.: Manufacturing of highly-dispersive, high-efficiency transmission gratings by laser interference lithography and dry etching. Microelectron. Eng. 191, 60-65 (2018)

63. Latourte, F., Rupin, N., He, M., Parrot, A., Leclercq, S.: Full field measurements used for assessing industrial issues-Two examples (2012) 
64. Lei, Z., Kang, H., Reyes, G.: Full field strain measurement of resistant spot welds using 3D image correlation. Exp. Mech. 50, 111-116 (2010)

65. Lindner, D., Mathieu, F., Hild, F., Allix, O., Ha Minh, C., Paulien-Camy, O.: On the evaluation of stress triaxiality fields in a notched titanium alloy sample via integrated DIC. J. Appl. Mech. 82(7), 071014 (2015)

66. Madeo, A., Barbagallo, G., d'Agostino, M., Placidi, L., Neff, P.: First evidence of non-locality in real band-gap metamaterials: determining parameters in the relaxed micromorphic model. Proc. R. Soc. A, vol. 472, p. 20160169. The Royal Society (2016)

67. Madeo, A., Neff, P., Ghiba, I., Placidi, L., Rosi, G.: Band gaps in the relaxed linear micromorphic continuum. arXiv preprint arXiv:1405.3493 (2014)

68. Madeo, A., Placidi, L., Rosi, G.: Towards the design of metamaterials with enhanced damage sensitivity: second gradient porous materials. Res. Nondestr. Eval. 25(2), 99-124 (2014)

69. Maier, G., Garbowski, T., Novati, G.: Practical advantages of full-field measurements and proper orthogonal decomposition for diagnostic analysis of dams and for free-foils characterization. In: Espinosa, H., Hild, F. (eds.) Full field measurements and identification in Solid Mechanics (Abstract Booklet) (2011)

70. Mathieu, F., Leclerc, H., Hild, F., Roux, S.: Estimation of elastoplastic parameters via weighted FEMU and integrated-DIC. Exp. Mech. 55(1), 105-119 (2015)

71. Maugin, G.A.: Generalized continuum mechanics: What do we mean by that?. In: Mechanics of Generalized Continua, pp. 3-13. Springer (2010)

72. Mindlin, R.: Force at a point in the interior of a semi-infinite solid. Physics 7(5), 195-202 (1936)

73. Mindlin, R., Tiersten, H.: Effects of couple-stresses in linear elasticity. Arch. Ration. Mech. Anal. 11(1), 415-448 (1962)

74. Mindlin, R.D.: Second gradient of strain and surface-tension in linear elasticity. Int. J. Solids Struct. 1(4), 417-438 (1965)

75. Misra, A., Lekszycki, T., Giorgio, I., Ganzosch, G., Müller, W.H., dell'Isola, F.: Pantographic metamaterials show atypical poynting effect reversal. Mech. Res. Commun. 89, 6-10 (2018)

76. Neggers, J., Hoefnagels, J., Hild, F., Roux, S., Geers, M.: A global digital image correlation enhanced full-field bulge test method. In: Espinosa, H., Hild, F. (eds.) Full field measurements and identification in Solid Mechanics, vol. Procedia IUTAM, 4, pp. 73-91. Elsevier, New York (2012)

77. Passieux, J., Périé, J.: Digital image correlation using proper generalized decomposition: PGD-DIC. Int. J. Numer. Methods Eng. 92(6), 531-550 (2012)

78. Passieux, J.C., Bouclier, R., Périé, J.N.: A Space-Time PGD-DIC Algorithm: application to 3D mode shapes measurements. Exp. Mech. (2018). https://doi.org/10.1007/s11340-018-0387-2

79. Pierron, F., Sutton, M., Tiwari, V.: Ultra high speed dic and virtual fields method analysis of a three point bending impact test on an aluminium bar. Exp. Mech. 51(4), 537-563 (2011)

80. Placidi, L., Andreaus, U., Giorgio, I.: Identification of two-dimensional pantographic structure via a linear d4 orthotropic second gradient elastic model. In: Journal of Engineering Mathematics, pp. 1-21 (2016)

81. Placidi, L., Barchiesi, E., Della Corte, A.: Identification of two-dimensional pantographic structures with a linear d4 orthotropic second gradient elastic model accounting for external bulk double forces. In: Mathematical modelling in solid mechanics, pp. 211-232. Springer (2017)

82. Placidi, L., Greco, L., Bucci, S., Turco, E., Rizzi, N.: A second gradient formulation for a $2 \mathrm{~d}$ fabric sheet with inextensible fibres. Zeitschrift für angewandte Mathematik und Physik 67(5), 114 (2016)

83. Rahali, Y., Giorgio, I., Ganghoffer, J., dell'Isola, F.: Homogenization à la piola produces second gradient continuum models for linear pantographic lattices. Int. J. Eng. Sci. 97, 148-172 (2015)

84. Ranaivomiarana, N., Irisarri, F.X., Bettebghor, D., Desmorat, B.: Concurrent optimization of material spatial distribution and material anisotropy repartition for two-dimensional structures. Continuum Mech. Thermodyn. 31(1), 133-146 (2019)

85. Réthoré, J., Limodin, N., Buffière, J., Roux, S., Hild, F.: Three-dimensional analysis of fatigue crack propagation using X-ray tomography, digital volume correlation and extended finite element simulations. In: Espinosa, H., Hild, F. (eds.) Full Field Measurements and Identification in Solid Mechanics, vol. Procedia IUTAM, 4, pp. 151-158. Elsevier, New York (2012)

86. Réthoré, J., Roux, S., Hild, F.: From pictures to extended finite elements: Extended digital image correlation (x-dic). C. R. Mécanique 335, 131-137 (2007)

87. Réthoré, J., Tinnes, J., Roux, S., Buffière, J., Hild, F.: Extended three-dimensional digital image correlation (x3d-dic). C. R. Mécanique 336, 643-649 (2008)

88. Rosi, G., Auffray, N.: Anisotropic and dispersive wave propagation within strain-gradient framework. Wave Motion 63, 120-134 (2016)

89. Roux, S., Hild, F., Leclerc, H.: Mechanical assistance to dic. In: Espinosa, H., Hild, F. (eds.) Full Field Measurements and Identification in Solid Mechanics, vol. Procedia IUTAM, 4, pp. 159-168. Elsevier, New York (2012)

90. Scerrato, D., Zhurba Eremeeva, I., Lekszycki, T., Rizzi, N.: On the effect of shear stiffness on the plane deformation of linear second gradient pantographic sheets. ZAMM-Journal of Applied Mathematics and Mechanics/Zeitschrift für Angewandte Mathematik und Mechanik (2016)

91. Sciarra, G., dell'Isola, F., Coussy, O.: Second gradient poromechanics. Int. J. Solids Struct. 44(20), 6607-6629 (2007)

92. Seppecher, P., Alibert, J.J., Isola, F.D.: Linear elastic trusses leading to continua with exotic mechanical interactions. In: Journal of Physics: Conference Series, vol. 319, p. 012018. IOP Publishing (2011)

93. Sun, Q.: Investigation of material heterogeneity in non-equilibrium solid-solid phase transition by full-field measurement method. In: Espinosa, H., Hild, F. (eds.) Full field measurements and identification in Solid Mechanics (Abstract Booklet) (2011)

94. Tanguy, A., Gounelle, M., Roux, S.: From individual to collective pinning: Effect of long-range elastic interactions. Phys. Rev. E 58, 1577-1590 (1998)

95. Tartar, L.: The General Theory of Homogenization: A Personalized Introduction, vol. 7. Springer, Berlin (2009)

96. Toupin, R.A.: Theories of elasticity with couple-stress. Arch. Ration. Mech. Anal. 17(2), 85-112 (1964) 
97. Turco, E., dell'Isola, F., Cazzani, A., Rizzi, N.: Hencky-type discrete model for pantographic structures: numerical comparison with second gradient continuum models. Zeitschrift für angewandte Mathematik und Physik 67 (2016)

98. Turco, E., Golaszewski, M., Giorgio, I., D’Annibale, F.: Pantographic lattices with non-orthogonal fibres: Experiments and their numerical simulations. Compos. B Eng. 118, 1-14 (2017)

99. Turco, E., Misra, A., Pawlikowski, M., dell'Isola, F., Hild, F.: Enhanced piola-hencky discrete models for pantographic sheets with pivots without deformation energy: numerics and experiments. In: International Journal of Solids and Structures (2018)

100. Yang, H., Ganzosch, G., Giorgio, I., Abali, B.E.: Material characterization and computations of a polymeric metamaterial with a pantographic substructure. Zeitschrift für angewandte Mathematik und Physik 69(4), 105 (2018)

101. Yildizdag, M.E., Tran, C.A., Barchiesi, E., Spagnuolo, M., dell'Isola, F., Hild, F.: A multi-disciplinary approach for mechanical metamaterial synthesis: A hierarchical modular multiscale cellular structure paradigm. In: State of the Art and Future Trends in Material Modeling, pp. 485-505. Springer (2019)

102. Yu, W., Tang, T.: Variational asymptotic method for unit cell homogenization. In: Advances in Mathematical Modeling and Experimental Methods for Materials and Structures, pp. 117-130. Springer (2009)

Publisher's Note Springer Nature remains neutral with regard to jurisdictional claims in published maps and institutional affiliations. 